\title{
Better Conversations with Aphasia: What are the interactional challenges of Wernicke's aphasia and how do people deal with them?
}

Suzanne Beeke ${ }^{1}$, Jane Maxim ${ }^{1}$, Claudia Bruns ${ }^{1}$, Fiona Johnson ${ }^{1,2}$, Firle Beckley ${ }^{1}$, Nicola Sirman ${ }^{3}$, Susan Edwards $^{1,4}$ \& Wendy Best ${ }^{1}$

1. Division of Psychology and Language Sciences, University College London, UK

2. Homerton University Hospital NHS Foundation Trust, London, UK

3. Solent NHS Trust, Southampton, UK

4. School of Psychology and Clinical Language Sciences, University of Reading, Reading, UK

Corresponding author s.beeke@ucl.ac.uk

Background: People with Wernicke's and other fluent aphasias often have severe and entrenched communication problems resulting from impaired comprehension and fluent yet semantically empty speech with neologisms and jargon. Impaired self-monitoring, leading to failure to correct speech errors is seen as a key challenge to successful communication. Speech and language therapists and key communication partners (CPs) report to us informally of the need for effective communication support for this client group. People with Wernicke's aphasia are considered unsuitable for many interventions, however there is some evidence of adaptive strategy use (Panzeri et al, 1987) and a few studies reveal awareness of speech errors in conversation (Laakso, 2003). This suggests there is conversation rehabilitation potential. This study reports on ongoing work to adapt the intervention techniques and materials of Better Conversations with Aphasia (BCA; Beeke et al, 2013) for speakers with Wernicke's and other severe fluent aphasias and their CPs.

Aims: To investigate the interactional challenges and adaptive strategies evident in family conversations of people with Wernicke's and other severe fluent aphasias. To use this understanding to advise on the use of conversation therapy for these speakers and their CPs.

Methods \& Procedures: Five people with Wernicke's aphasia (as ascertained using the Western Aphasia Battery) were recruited via successive projects carried out by student speech and language therapists at UCL. Ethical approval for this programme of work was granted by the Language \& Cognition Departmental Ethics Chair on behalf of the Division of Psychology \& Language Sciences. The person with aphasia and a familiar CP were asked to video record at least 20 minutes of conversation in their home environment. No topics were set or suggested, participants were encouraged to video record at a time when they had new information to share, for example when catching up about the day's events. The data were analysed using Conversation Analysis (CA), a qualitative sociolinguistic method for the systematic examination of interactional strategies and challenges. Patterns of interactional behaviour were sought across each dyad, followed by analysis of commonalities across dyads.

Outcomes \& Results: CA revealed common conversational barriers around repeated and often unsuccessful attempts at self-repair of speech errors. One particularly complex pattern involves delayed self-repair initiation and ambiguity around when or if repair is completed. Thus, ' $X$ ' is repeated on multiple occasions before rejection in the form of 'not $X$ ' occurs, often after the conversation appears to have continued via intervening talk by the person with aphasia or the CP. Sometimes the expected replacement (' $Y$ ') is not spoken, or it is hard to identify which of the successive words spoken is the replacement for the rejected $X$. This is illustrated in Extract 1. 
Adaptive behaviours by the person with aphasia include explicit comments on word finding difficulty, and minimisation of attempts to repair errors when engaged in story-telling sequences over multiple turns, perhaps to facilitate the flow of the story. CP barriers to conversation include therapeutic-like cueing of words in the midst of multiple attempts at a target by the person with aphasia; these did not help and sometimes actively derailed the conversation. The data also reveal spontaneous adaptive strategies by a CP including 'so'-prefaced understanding checks after long turns at talk by a person with aphasia. Some of these behaviours appear different from the predominant barriers and facilitators to conversation in non-fluent aphasia, such as the repeated attempts at self-repair with no clear indication of whether the intended word has been found, and the 'so'-prefaced understanding checks. However, the use of 'test' questions by a CP appears to be a universal pattern.

Conclusions: Based on what we have learned from evaluating the BCA intervention programme for people with non-fluent aphasia, it is possible to identify interactional behaviours with the potential to change after conversation training for people with Wernicke's and other severe fluent aphasias and their CPs. Preliminary findings suggest that people with this aphasia type who show awareness of the need to self-correct speech errors, and who comment on their word finding difficulties, may have the potential to respond to direct conversation training alongside a CP. A next step will be to test this out directly by evaluating conversation training with dyads where one speaker has severe fluent aphasia.

References

Beeke S, Sirman N, Beckley F, Maxim J, Edwards S, Swinburn K, Best W. (2013). Better Conversations with Aphasia: an e-learning resource. Available at: https://extend.ucl.ac.uk/

Laakso, M. (2003) Collaborative construction of repair in aphasic conversation: An interactive view on the extended speaking turns of persons with Wernicke's aphasia. In C. Goodwin (Ed.), Conversation and Brain Damage. Oxford: Oxford University Press.

Panzeri, M., Semenza, C., \& Butterworth, B. (1987). Compensatory processes in the evolution of severe jargon aphasia. Neuropsychologia 25(6), 919-933. http://doi.org/10.1016/0028-

3932(87)90096-0 